\title{
A Novel Technique for Improving Hardware Trojan Detection and Reducing Trojan Activation Time
}

\author{
Hassan Salmani and Mohammad Tehranipoor \\ ECE Department \\ University of Connecticut \\ \{salmani_h,tehrani\}@engr.uconn.edu
}

\author{
Jim Plusquellic \\ ECE Department \\ University of New Mexico \\ jimp@ece.unm.edu
}

\begin{abstract}
Fabless semiconductor industry and government agencies have raised serious concerns about tampering with inserting hardware Trojans in IC supply chain in recent years. Most of the recently proposed Trojan detection methods are based on Trojan activation to observe either a faulty output or measureable abnormality on side-channel signals. Time to activate a hardware Trojan circuit is a major concern from the authentication standpoint. This paper analyzes time to generate a transition in functional Trojans. Transition is modeled by geometric distribution and the number of clock cycles required to generate a transition is estimated. Furthermore, a dummy scan flip-flop insertion procedure is proposed aiming at decreasing transition generation time. The procedure increases transition probabilities of nets beyond a specific threshold. The relation between circuit topology, authentication time, and the threshold is carefully studied. The simulation results on s38417 benchmark circuit demonstrate that, with a negligible area overhead, our proposed method can significantly increase Trojan activity and reduce Trojan activation time.
\end{abstract}

Index Terms - Security, Hardware Trojan, Trojan Detection, Trojan Activation Time, Dummy Flip-Flop Insertion

\section{INTRODUCTION}

Outsourcing design and fabrication process has become a trend in Integrated Circuit (IC) market due to economical profit, with limiting the control of customer over IC supply chain. Motivated adversary takes advantage of such restriction to tamper IC supply chain by maliciously implanting extra logic as hardware Trojan circuitry into an IC [1]. Consequently serious concerns rise about security and trustworthiness of electronic systems. An attacker can change a design netlist or subvert the fabrication process by manipulating design mask, without affecting the main functionality of the design [2].

Hardware Trojan detection is an extremely challenging problem and traditional structural and functional tests cannot effectively address it. Trojan circuits have stealthy nature and are triggered in rare conditions. Trojans are designed such that they are silent most of their life time and may have very small size relative to their host design, with featuring limited contribution into design characteristics. These suggest that they most likely connect to nets with low controllability and/or observability [3][4][5]. It is expected that Trojan inputs are supplied by nets with low transition probabilities to lessen its impact on circuit side-channel signals such as power and delay. Automatic Test Pattern Generation (ATPG) methods used in manufacturing test for detecting defects do so by operating on the netlist of the Trojan-free circuit. Therefore, existing ATPG algorithms cannot target Trojan directly [3].

Trojan detection makes efficient pattern generation necessary to disclose Trojan impact on design characteristics beyond process and environmental variations. Trojan detection methods using transient power analysis [6][7][8][9] require patterns that increase Trojan activity whereas keep circuit activity low to magnify Trojan contribution into the circuit power consumption. Methods that are based on delay analysis [10] [11] require patterns that generate transition on nets that supply Trojan inputs to reveal wiring and input gate resistance and capacitance impact of Trojan on the circuit delay characteristic. From authentication standpoint, it is critical to (i) analyze time to generate a transition at Trojan input and in Trojan circuit and (ii) reduce authentication time.

In this paper, we develop a methodology to increase the probability of generating a transition in functional Trojan circuits and to analyze the transition generation time. Transition probability is modeled using Geometric Distribution [12] and is used to estimate number of clock cycles required to generate a transition on a net. An efficient dummy flip-flop insertion procedure is proposed to remove rare triggering condition of Trojans. The procedure identifies nets with transition probability less than a specific transition probability and inserts dummy flip-flops such that the transition probabilities of all nets in design are greater than the specific transition probability. It should be noted that dummy flip-flops are inserted in a way that will not change the functionality and timing of design. The effectiveness of dummy flip-flop insertion is examined by evaluating different transition probability thresholds for various Trojan circuits. The relation between authentication time, the number of required transitions in Trojan circuit, and tester clock is studied. These parameters would help determine the transition probability threshold of a design. The transition probability threshold, in turn, provides an estimation of area overhead induced by inserted dummy flip-flops. Our simulation results show significant improvement in Trojan detection and reduction in Trojan activation time.

The paper is organized as following: Section II describes prior work on Trojan detection. Analyzing Trojan activation time is presented is Section III. The proposed dummy flipflop insertion procedure is presented in Section IV. Transition probability threshold analysis and simulation results are presented in Sections V and VI. Finally the concluding remarks are presented in Section VII. 


\section{PRIOR WORK}

In [4], the authors present a sustained vector technique. A vector is applied to circuit and for several clock cycles (up to 25) primary inputs are kept unchanged. In this way all transitions in the circuit would be attributed to state bits and it is expected that activities converge to a specific portion of the circuit after some clock cycles. By applying the next vector another portion of the circuit will be targeted.

Authors in [8] present a method to generate a power fingerprint of genuine ICs considering various types of noise in the circuit. Random patterns are applied to IC-UnderAuthentication (IUA) to generate a measurable difference between the power profiles of the genuine IC and IUA.

The proposed method in [9] is based on analyzing local $I_{D D T}$ current measured from power ports on the target chip. A calibration process is performed for each IUA before actual measurement to alleviate process variations impact. Trojaninserted designs are distinguished using outlier analysis. In [13], a multiple supply transient current integration method is presented to detect hardware Trojans in IUA. The current is measured locally from various power pads or controlled collapse chip connections $(\mathrm{C} 4 \mathrm{~s})$ on the die. Random patterns are applied to increase the switching in the circuit in a testper-clock fashion [14].

A randomization based probabilistic approach to detect Trojans is presented in [15]. The authors show that it is possible to construct a unique probabilistic signature for each circuit by applying input patterns with specific probability. The output of IUA after applying each pattern is compared with that of golden circuit. In case of any difference, Trojan infection is reported. Otherwise, after applying a set of $N$ vectors if the IUA and golden circuit give the same output, using statistical reasoning, it is reported that the IUA is Trojanfree with a confidence interval.

Gate-level characteristics can be used to detect hardware Trojans [16][17]. Delay and power characteristics of each gate of a design subjected to process variation can be individually extracted. Linear programming is used to solve a system of equations created using non-destructive measurements of power and delays. Any extra power consumption or excessive delay caused by Trojan may manifest in measurement error or new characteristics for circuit's gates.

A comprehensive taxonomy of Trojans in integrated circuits is presented in [3]. Trojans are classified based on physical, activation, and action characteristics. The physical characteristic studies type, size, distribution, and structure of a Trojan. In terms of type, Trojan can be functional or parametric. Functional Trojans are realized through adding or deleting of transistors or gates, while parametric ones are realized through modification of physical geometry of design to sabotage reliability. The number of gates or transistors which are added or deleted defines Trojan size. Distribution refers to the locations of Trojan components in physical layout. They can be tight (i.e. placed close to each other) or loose (i.e. dispersed across the layout.) Trojan insertion can affect chip dimension, delay characteristic and power profile of a circuit.
Trojan activation characteristics refer to criteria that cause Trojan to activate and carry out its disruptive function. The types of disruptive behavior introduced by Trojan determines Trojan action characteristics. For more details on Trojan taxonomy, reader is referred to [3]. In this work, we focus on functional Trojans and targeting parametric Trojans will be part of our future work.

\section{Trojan Activation Time Analysis}

Since there is no information about Trojan circuit in terms of size, type, or location, from authentication standpoint, it is crucial to analyze Trojan activation time (partially or fully). In this paper, full activation of Trojan refers to patterns that activate Trojan so that it impacts the circuit output and causes malfunction. However, partial activation refers to generating one or more transitions inside Trojan circuit so that it improves the effectiveness of transient power-based methods [8][9][13]. In general, a functional Trojan consists of two parts: Trigger and Payload [18]. The Trigger circuit is mostly inactive by nature with no Payload effect. Under certain rare conditions or events, the Trojan is activated (triggered) and then Payload injects an error to the circuit. Generating transition in Trojan circuit depends on its implementation. Switching at the first level gates of Trojan circuit depends on its preceding cells. The next levels of Trojan circuit are similar to the first level; therefore, in the following, we focus on generating switching in one Trojan gate at the first level of a Trojan circuit to carry out our detailed analysis. However, the results in Section VI will be presented for the entire Trojan circuit.

In general, the transitions in a circuit are induced by transitions in scan cells and primary inputs [20]. We define a Trojan cone as logic circuit connecting to the inputs of a Trojan gate. Note that, in this section, we present one Trojan gate for our analysis; however, a Trojan may contain more than one gate. Also, note that we do not assume the location of Trojan is known to us. The procedure developed in this work is independent of location and size of hardware Trojan in integrated circuits. Trojan cone can determine the required time to generate transition in a Trojan gate. The number of gates, gate types and the structure of Trojan cone can define time to generate transition in the Trojan gate as well. Figure 1 shows two example Trojan cones. Trojans are named as Trojan 1 and Trojan 2. Trojan 1 contains three gates in two levels while Trojan 2 contains seven gates in three levels. $T_{g 1}$ in Trojan 1 is connected to the cone shown in Figure 1(a) and $T_{g 3}$ is connected to the cone in Figure 1(b). Other gates in the two Trojans are assumed to be connected to other parts of the circuit.

In Figure 1(a), Trojan cone consists of 17 gates in 11 levels. Trojan cone contains all gates in original circuit impacting a Trojan gate and the Trojan gate itself (here $T_{g 1}$ ). Simulation results show that after applying 1000 random test vectors in test-per-clock fashion, there are 67 transitions at $T_{g 1}$ output.

In Figure 1(b), Trojan cone consists of 7 gates in 2 levels. The simulation results show that there are 421 transitions at $T_{g 3}$ output after applying the same number of test vectors, i.e. 


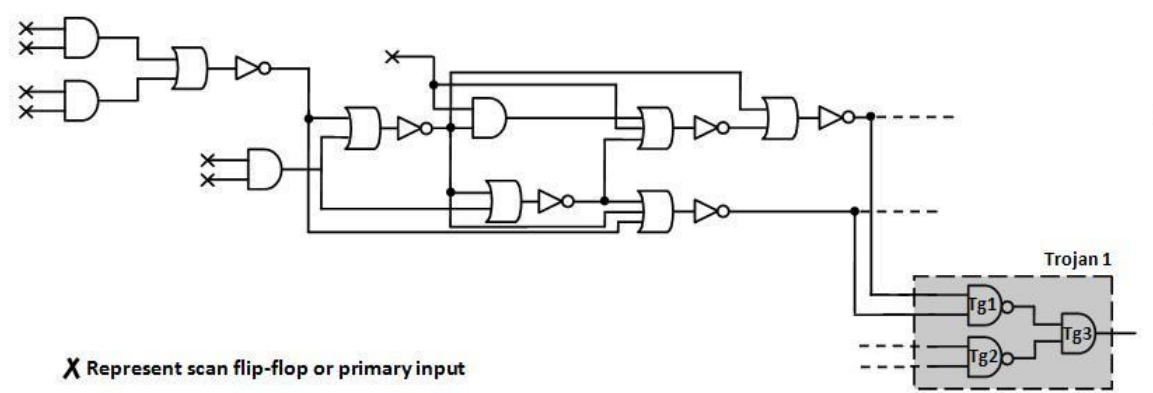

(a)

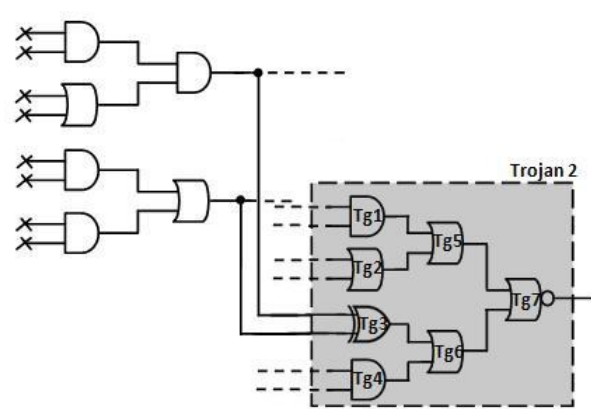

(b)

Fig. 1. Two Trojan cone examples: (a) Trojans 1 and (b) Trojan 2

1000. Since random vectors are applied to the above circuits, the results can be slightly different from one random vector set to another. As seen from the results, the number of transitions in the two Trojan gates varies significantly. This is mainly due to the difference in Trojan cone structure, number of levels, and number of inputs (scan flip-flops and primary inputs) and Trojan gate type.

Probability can represent characteristics of a circuit since it considers gates functionality and interconnections among them. The probability of switching at a node in the circuit provides a good estimation of the time to generate switching on the node. Trojan cone determines switching probability at the Trojan gate output, e.g. $T_{g 1}$. Suppose the probabilities of having ' 1 ' and ' 0 ' at Trojan output are $P 1$ and $P 0$, respectively, the probability of switching from 0 to 1 or 1 to 0 at the output of a Trojan gate will be $P t_{T g i}=P 1 \times P 0$, where $T_{g i}$ is the $i$ th gate at the first level of a Trojan. For example, with assumption of applying random patterns through inputs, with probability of $1 / 2$, the probability of generating a transition at the output of Trojan gate $T_{g 3}\left(P t_{T g}\right)$ is 0.25 as shown in Figure 2. The circuit shown in this figure is the same as one depicted in Figure 1(b).

To obtain transition probability, a transition (i.e. success) can be modeled using Geometric Distribution (GD) [12]. The Geometric Distribution is a discrete distribution for $n=$ $0,1,2, \cdots$ with the probability function $p(n)=P \times(1-P)^{n}$. The probability function states that after $n$ clock cycles, finally in the $(n+1)$ th clock cycle, there will be a transition, i.e. $(n+1)$ th trial is the first success. The average number of experiments is $\left(P^{-1}-1\right)$ which indicates the number of required clock cycles, on average, to generate a transition.

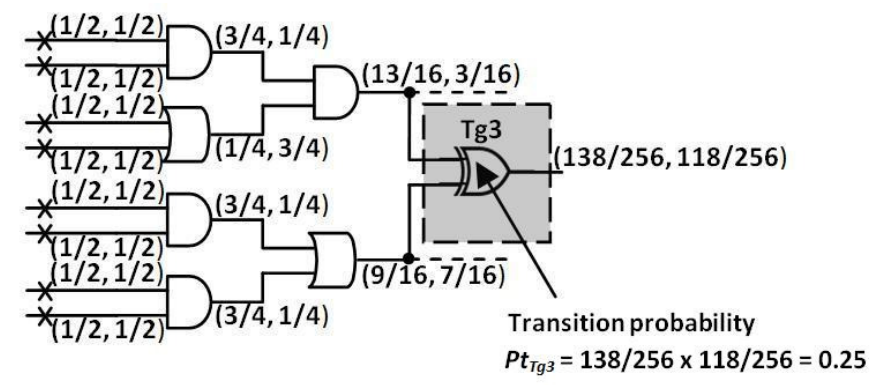

$X$ Represents scan flip-flop or primary input

Fig. 2. Transition probability for a target cone.

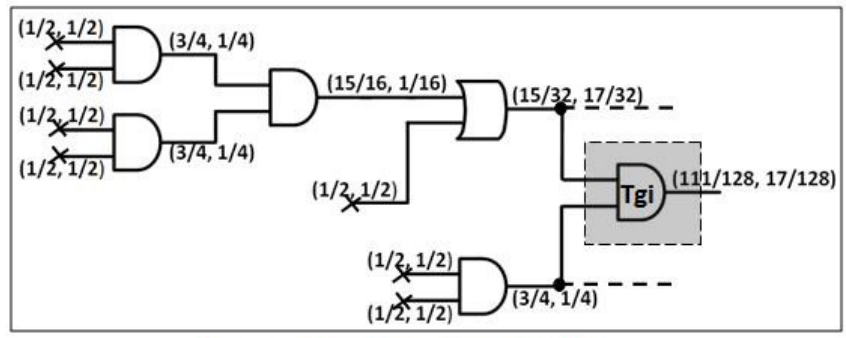

Transition probability at Trojan output $=1887 / 16384$ Average clock cycles per transition by $\mathrm{GD}=\mathbf{7 . 6 8}$ Average clock cycles per transition by simulation $=7.46$

(a)

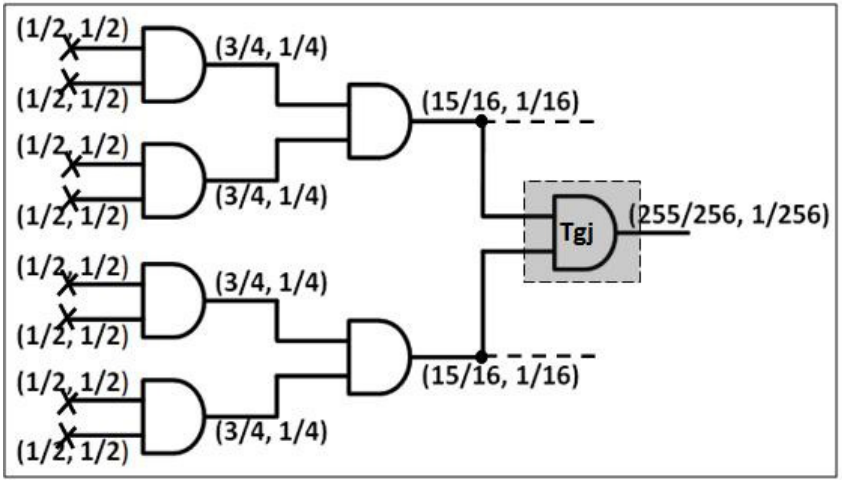

Transition probability at Trojan output $=255 / 65536$

Average clock cycles per transition by GD $=255.6$

Average clock cycles per transition by simulation $=250$

(b)

$X$ Represent scan flip-flop or primary input

Fig. 3. Comparing mathematical and simulation results.

For the Trojan gate shown in Figure 2, the calculation based on Geometric Distribution shows that on average three clock cycles are required to generate a transition at the Trojan gate $\left(T_{g 3}\right)$ output. This is demonstrated by our simulation results since, on average, in each 2.37 clock cycles a transition was generated after applying 1000 test vectors. Note that the 1000 random test vectors are generated with the probability of $1 / 2$ for ' 0 ' and ' 1 '.

Figure 3 presents two new Trojan cones and compares the average clock cycles per transition using GD (i.e. probability analysis) and simulation. Figure 3(a) shows that the simulation result of applying 1000 random patterns is very close to that of GD. Trojan cone in Figure 3 consists only of AND gates such that the probability of generating ' 1 ' at Trojan gate $T_{g j}$ output is much less than that of ' 0 ' therefore, there is a 
TABLE I

S298 AND S344 BENCHMARKS CHARACTERISTICS.

\begin{tabular}{|l||l|l|l|l|}
\hline Benchmark & $\begin{array}{l}\text { \# of in- } \\
\text { puts }\end{array}$ & $\begin{array}{l}\text { \# of flip- } \\
\text { flops }\end{array}$ & $\begin{array}{l}\text { the total number } \\
\text { of inputs and flip- } \\
\text { flops }\end{array}$ & $\begin{array}{l}\text { the number } \\
\text { of gates }\end{array}$ \\
\hline s298 & 3 & 14 & 17 & 68 \\
\hline s344 & 9 & 15 & 24 & 71 \\
\hline
\end{tabular}

small transition probability for $T_{g j}$. Any transition to ' 1 ' will most likely follow immediately by a transition to ' 0 ' since Trojan cone mostly provides ' 0 ' at the output of $T_{g j}$ gate. The simulation results by applying 1000 test vectors show that each 250 clock cycles there is one transition at Trojan output and probability analysis shows that every 255.6 clock cycles, one transition can be generated at the output of $T_{g j}$ gate.

Beside the interconnection among gates (i.e. circuit topology), transition probabilities of nets depend on the number of inputs and flip-flops of Trojan cone. Primary inputs and flipflops can determine a net's depth which is the minimum distance of the net from either a primary input or a flip-flop. Such dependency is examined on two ISCAS' 89 benchmarks (s298 and s344). Table I shows the benchmarks' characteristics. The benchmarks have roughly the same number of gates; however, their number of inputs and flip-flops are different. Primary inputs and flip-flops provide immediate access to internal parts of a circuit, and thus increase transition probabilities of nets. Figure 4 compares the transition probabilities frequency of the benchmarks.

Figure 4 shows that $\mathrm{s} 344$ benchmark, having more number of inputs and flip-flops, has more number of nets with high transition probability. Further, simulation results of applying random vectors in 1000 clock cycles report 56560 transitions in s344 while 44600 in s298. Therefore, enhancing accessibility to internal parts of circuit by inserting dummy flip-flops can be an effective way to increase transition probability of nets.

It is seen from both analyses (GD and simulation) that as $P 0$ or $P 1$ of a net becomes too large or too small, the transition probability reduces significantly. Therefore, to increase transition probability of a net, it would be preferred to ensure that $P 0$ and $P 1$ values are close. The maximum transition probability of a net can be $P t=0.25$ and it happens when $P 0=P 1=1 / 2$. Given a cone structure and various gate types used in the cone, making the transition probability

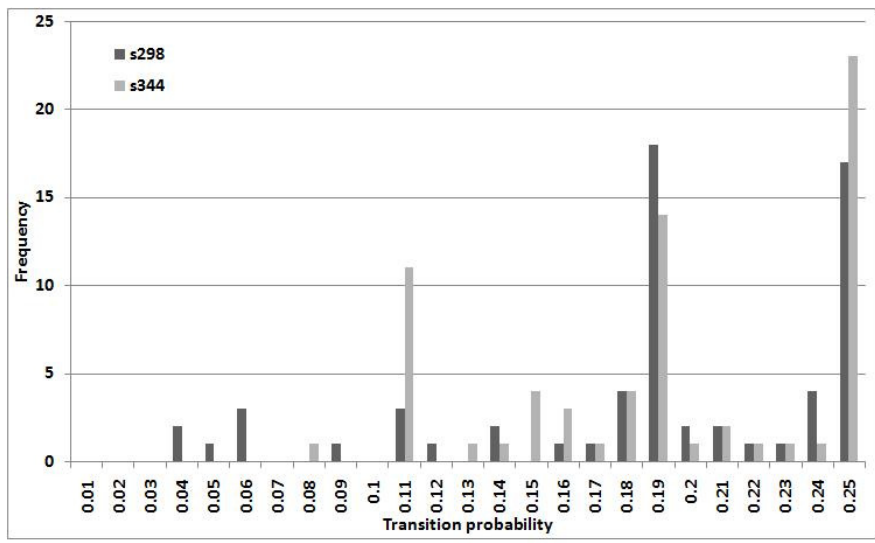

Fig. 4. Transition probability frequency in s298 and s344 benchmarks.

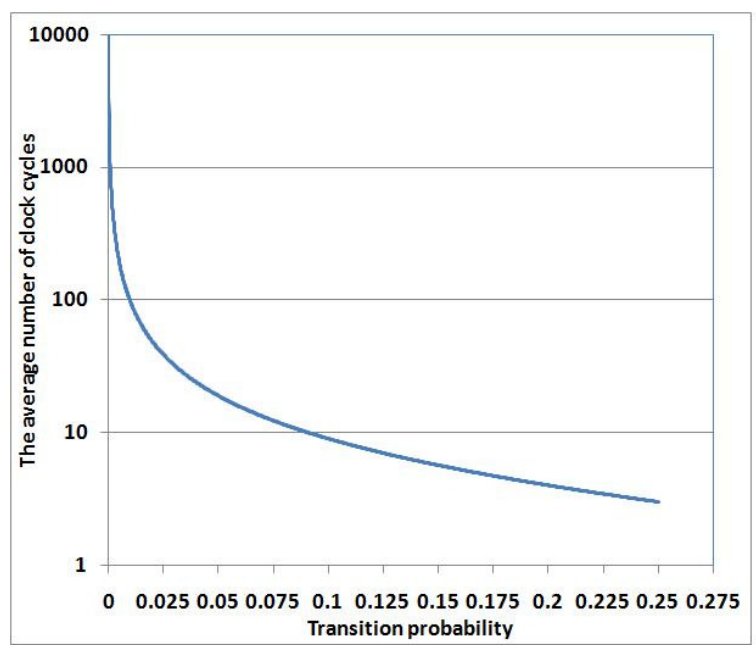

Fig. 5. The number of required clock cycles versus transition probability based on geometric distribution.

values $(P t)$ closer to each other would seem impractical but by improving controllability by inserting dummy flip-flops, we would be able to increase transition probability for both $0 \rightarrow 1$ and $1 \rightarrow 0$ transitions. This is validate by the analysis performed using Geometric Distribution as shown in Figure 5. As seen, as the transition probability decreases, the number of clock cycles to generate transition increases exponentially.

\section{DUMmy SCAN FLIP-FLOP INSERTION}

When the probabilities for ' 1 ' and ' 0 ' of nets on a path in a cone becomes unidirectional, i.e. $P 1 \gg P 0$ or $P 0 \gg$ $P 1$ similar to the example shown in Figure 3(b), transition probability of the nets $\left(P t_{i}=P_{i} 0 \times P_{i} 1\right)$ rapidly decreases. To ensure transition probabilities are greater than a specific threshold $\left(P_{T H}\right)$, dummy flip-flops can be inserted so as to close probabilities of ' 1 ' and ' 0 ' nets. Note that in this paper both terms "dummy flip-flop" and "dummy scan flip-flop" refer to the increased controllability (transition probability) in a circuit.

Figure 6 shows the structure of dummy scan flip-flop (dSFF) in addition to an extra gate (AND or OR). If probability of ' 0 ' on target net $N e t_{i}, P_{i} 0$, is less than its probability of ' 1 ', $P_{i} 1$, an AND gate is placed after scan flip-flop and net $N e t_{i}$ restitched through the AND gate to increase $P_{i} 0$, as depicted in Figure 6(a). However, if $P_{i} 1$ is less than $P_{i} 0$, an OR gate is being used to increase $P_{i} 1$, as in Figure 6(b). In this work, $d S F F-A N D$ and $d S F F-O R$ represent dummy scan flip-flops with AND and OR gates, respectively. Accompanying a net having low transition probability with a dSFF would increase the net's and following nets' transition probabilities. When

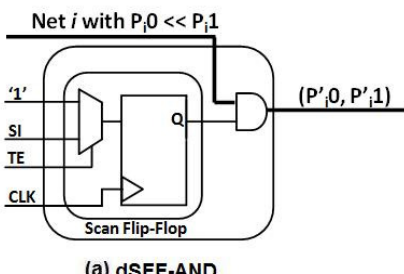

(a) dSFF-AND

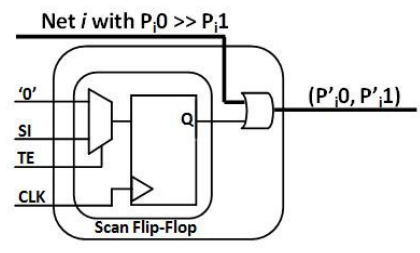

(b) dSFF-OR
Fig. 6. The dummy flip-flop structures when (a) $P_{i} 0 \ll P_{i} 1$ and (b) $P_{i} 0 \gg$ 
Test Enable (TE pin) is active, the output of scan flip-flop is supplied by Scan Input (SI pin). The inserted dummy scan flip-flop has no impact on the functionality of the circuit. In normal functional mode, the output of scan flip-flop is supplied by either ' 0 ' or ' 1 ' depending on the gate type at the output of scan flip-flop to avoid changing the functionality of $\mathrm{Net}_{i}$.

The probabilities of ' 1 ' and ' 0 ' at the output of scan flipflop are $1 / 2$. Thus, by supplying internal nets with nets having equal ' 1 ' and ' 0 ' probabilities, the ' 1 ' and ' 0 ' probabilities on target nets can become closer and their respective transition probabilities can be increased. Assume that $P_{i} 0$ of Net $_{i}$ is much greater than its $P_{i} 1$, where

$$
P_{i} 0=\frac{K}{N} \quad \text { and } \quad P_{i} 1=1-\frac{K}{N}
$$

where $K$ and $N$ are cardinal values. The denominators of probabilities would be the number of clock cycles in an experiment and their numerators are the number of desired value. $P_{i} 0$ approaches to 1 (i.e. $K \approx N$ ) when it is assumed $P_{i} 0 \gg P_{i} 1$. By inserting proposed dummy flip-flop as in Figure 6(b), new probabilities are

$$
P_{i}^{\prime} 0=\frac{K}{2 N}, \text { and } P_{i}^{\prime} 1=1-\frac{K}{2 N}
$$

As a result, $P_{i}^{\prime} 0$ will be smaller than $P_{i} 0$ and $P_{i}^{\prime} 1$ will be greater than $P_{i} 1$. Thus, after dummy flip-flop insertion, the transition probability of the target net and its following nets would be greater as

$$
\begin{gathered}
P_{i}^{\prime} 0 \times P_{i}^{\prime} 1>P_{i} 0 \times P_{i} 1 \\
\frac{K}{2 N} \times\left(1-\frac{K}{2 N}\right)>\frac{K}{N} \times\left(1-\frac{K}{N}\right) \\
2 N-K>4(N-K)
\end{gathered}
$$

which is true because $K \approx N$ and $N>K$.

Using same analysis, it can be proven that by inserting AND gate when $P_{i} 0$ of a net is much lower than its $P_{i} 1$, the transition probability of the net can be increased. In this case, mathematical analysis shows that inserting a dSFF-OR on upper input net of $T_{g i}$ gate in Figure 3, as depicted in Figure 7 , reduces the number of clock cycles per transition from 255.6 to 30 on average at the output of $T_{g j}$ gate. Furthermore, simulation results also closely confirm 33.4 clock cycles per transition.

\section{A. Removing Rare Triggering Conditions}

An able adversary would ensure that Trojans are activated only under very rare conditions. It could be a rare circuit state, certain temperature or noise, etc. This is necessary to avoid Trojan detection accidentally using structural or functional patterns. As an example, for functional Trojans [3], a Trojan can have $q \gg 1$ trigger inputs which can be nets with (i) very low transition probabilities and (ii) rare combinations. When the transition probability of $\mathrm{Net}_{i}$ is very low, either $P_{i} 0$ is much greater than $P_{i} 1$ or vice versa, as discussed in
Section III. With $q$ number of trigger inputs, the probability of generating a specific trigger vector is

$$
P_{\text {trigger-vector }}=\prod_{i=1}^{q} P_{i}
$$

where

$$
P_{i}= \begin{cases}P_{i} 0 & \text { for trigger input } N e t_{i} \text { to be } 0 \\ P_{i} 1 & \text { for trigger input } N e t_{i} \text { to be } 1 .\end{cases}
$$

It is expected that $P_{\text {trigger-vector }}$ to be very low if $P_{i} 0$ or $P_{i} 1$ is low. By inserting dummy scan flip-flop, the transition probability of nets would increase since $P_{i} 0$ and $P_{i} 1$ values become closer. As a result, $P_{\text {trigger-vector }}$ also increases and the trigger vector will not be a rare event anymore. By increasing the transition probability of nets with low transition rate, we will eliminate hard-to-activate sites in a design. This would result in increasing the probability of switching in Trojan circuit. If fully activated, Trojan's output can impact design functionality and it will be detected. In case of increasing switching in the Trojan, called partial activation in this paper, the Trojan can be detected much easier using transient power or charge-based analysis methods [8][9][13]. This method eliminates the need to focus on rare conditions as proposed in [18][19].

For example, Table II shows probability of two nets in s38417 benchmark before and after dummy scan flip-flop insertion. Assuming that Trojan needs trigger vector $\{01\}$ on Net 1 and Net2, as seen in the table, the probability of the trigger vector would be $P_{\text {trigger-vector }}=P_{N e t 1} 0 \times$ $P_{\text {Net2 } 2} 1=4.079 \mathrm{e}-06$ in the original circuit without dummy flipflop. However, the probability increases to 0.094 after dummy flip-flop insertion.

\section{B. Dummy Scan Flip-Flop Insertion Procedure}

Figure 8 shows the proposed dSFF insertion procedure. Nets with transition probabilities greater than determined transition probability threshold $\left(P_{T H}\right)$ and close to nets with transition probabilities lower than $P_{T H}$ are good candidates for dSFF insertion since each of them can impact several low transition nets at their fanout cone at once.

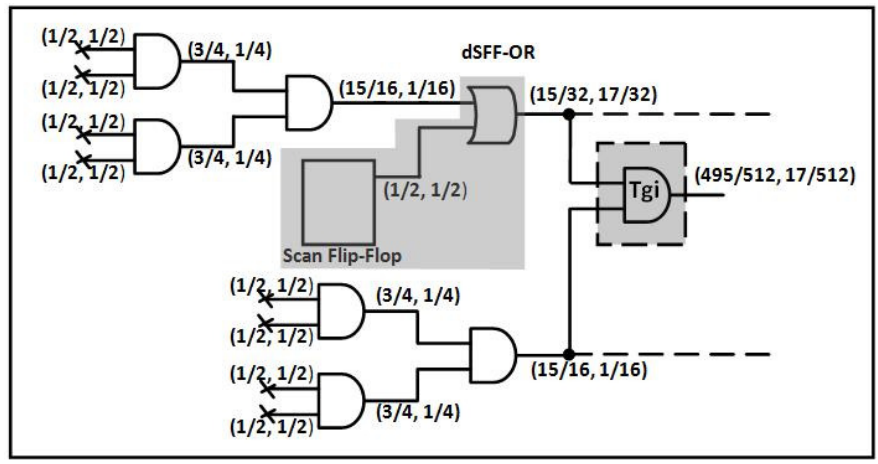

Transition probability at Trojan output $=8415 / 262177$

Average clock cycles per transition by GD $=\mathbf{3 0}$

Average clock cycles per transition by simulation $=\mathbf{3 3 . 4}$

$\Varangle$ Represent scan flip-flop or primary input

Fig. 7. Increasing transition probability by inserting dSFF-OR 
TABLE II

PROBABILITY OF TWO NETS IN S38417 BENCHMARK BEFORE AND AFTER DSFF INSERTION.

\begin{tabular}{|c|c|c|c|c|c|c|}
\hline & \multicolumn{3}{|c|}{ Before dSFF insertion } & \multicolumn{3}{|c|}{ After dSFF insertion } \\
\cline { 2 - 7 } & $P 0$ & $P 1$ & $P_{\text {Net } 10 \times P_{\text {Net } 2} 1}$ & $P 0$ & $P 1$ & $P_{\text {Net } 10 \times P_{\text {Net } 2} 1}$ \\
\hline \hline Net 1 & 0.999995317077 & $4.6 \mathrm{e}-06$ & $4.079 \mathrm{e}-06$ & 0.989 & 0.011 & 0.094 \\
Net 2 & 0.999959170737 & $4.08 \mathrm{e}-06$ & & 0.905 & 0.095 & \\
\hline
\end{tabular}

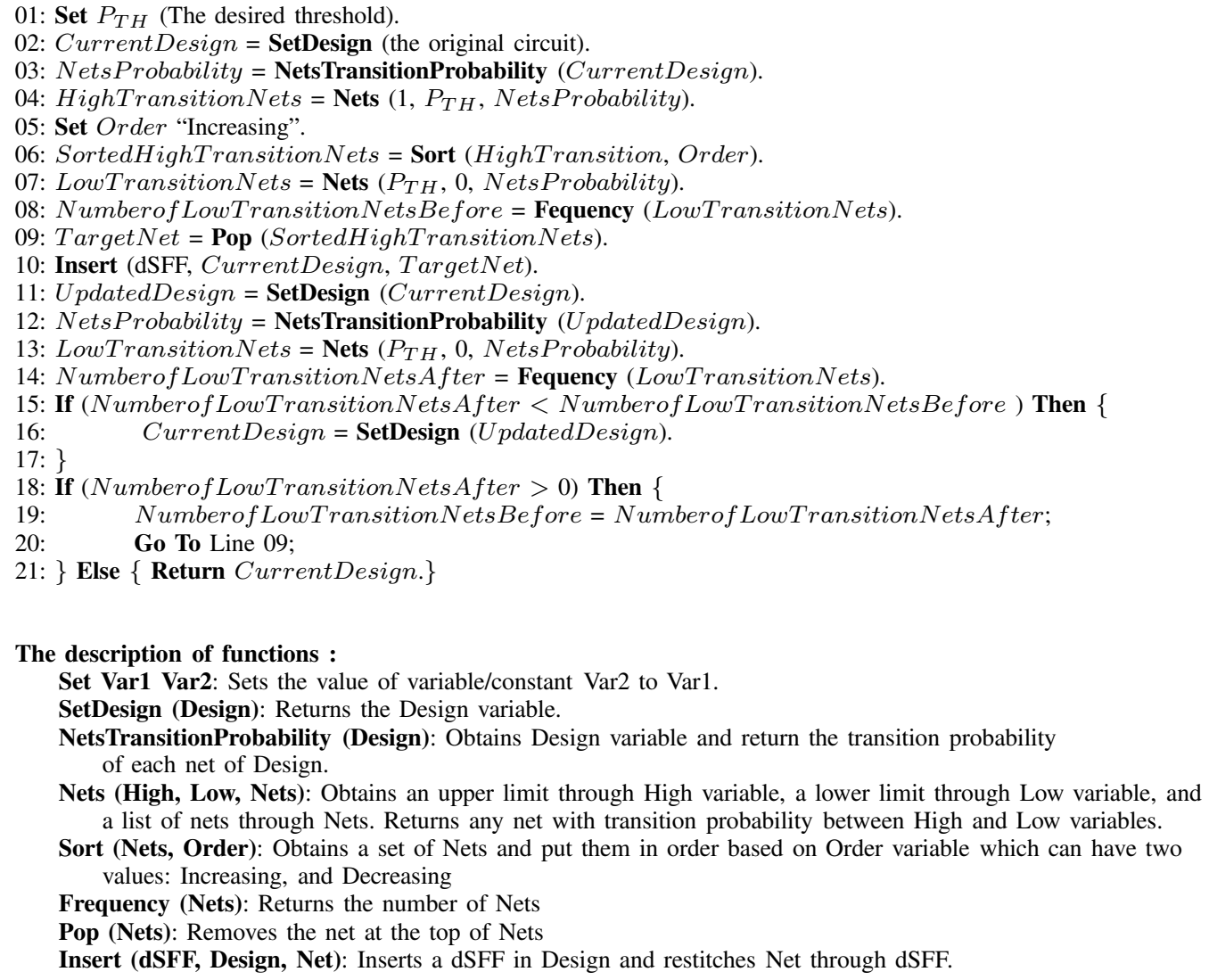

Fig. 8. dSFF insertion procedure.

After setting $P_{T H}$ and original design as CurrentDesign (Lines 1-2), the procedure will calculate transition probability of all nets in the design (Line 3). Nets are then divided into two groups: (1) nets with transition probability higher than $P_{T H}$, and (2) nets with transition probability lower than $P_{T H}$. Nets in the first group obtained in Line 4 are then sorted and permanently stored in SortedHighTransitionNets (Line 6).

In the following, in Lines 7 and 8, nets with transition probability less than $P_{T H}$ are identified and stored as LowTransitionNets and their number as NumberofLowTransitionNetsBefore. The procedure, in Line 9, removes the net with the lowest/highest transition probability, depending upon Order, from SortedHighTransitionNets. The removed net is restitched through dSFF in Line 10. Transition probability of nets after dSFF insertion is again calculated and the number of low transition nets is obtained. If the value is less than the number of low transition nets before dSFF insertion, the inserted dSFF is kept otherwise the dSFF would be ignored since no gain was obtained. In the following, if there is still any net with transition probability less than $P_{T H}$, the procedure continues until there would not be neither any net with low transition probability nor any nets in SortedHighTranstionNets.

We acknowledge that inserting dummy scan flip-flop increases the delay of paths and can impact design performance. Note that it is unlikely that adversary uses nets on critical paths as input since it can impact the path delay due to the increased capacitance and can be easily detected using path delay fault test patterns. Using the above procedure, we avoid inserting dummy flip-flops on critical paths by eliminating nets on the critical paths from HighTransitionNets.

\section{TRAnsition Probability Threshold Analysis}

Inserting dummy flip-flops to increase transition probability of nets would increase circuit area. The area overhead mainly depends on transition probability threshold $\left(P_{T H}\right)$. By setting a $P_{T H}$, our proposed procedure ensures that all nets in the circuit have transition probability greater or equal to this threshold. $P_{T H}$ would impact both area overhead (i.e. the number of 


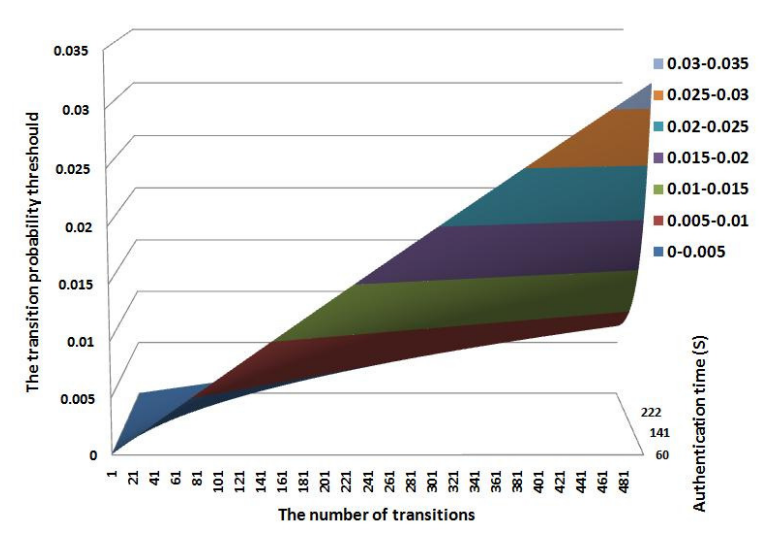

Fig. 9. Probability threshold versus authentication time and the number of transitions.

dSFFs) and transition generation time in hardware Trojan gates. In general, setting smaller $P_{T H}$ would result in smaller number of dSFFs but would require more time, on average, to generate switching in Trojan gates. On the other hand, setting larger $P_{T H}$ would require more number of dSFFs but reduces the transition generation time in hardware Trojan gates.

To set $P_{T H}$, there are several parameters that should be considered. They can be grouped into two main categories namely authentication and circuit parameters. Authentication parameters are of authentication characteristics and consist of two sub-parameters: 1) authentication time of each integrated circuit, $T_{A u}$, and 2) the clock period of tester, $T_{T e s t e r}$. Circuit parameters represent circuit characteristics and consist of three sub-parameters: 1) the number of required transitions in Trojan circuit, $\left.N_{T r}, 2\right)$ the average number of clock cycles per transition which can be modeled using Geometric Distribution, and 3) circuit activity, $C_{\text {activity }}$. Note that $N_{T r}$ is an important parameter when using transient power analysis methods for detecting hardware Trojans since it indicates the contribution of Trojans into the total circuit power consumption. The larger the $N_{T r}$, the easier the detection of a Trojan would be.

Equation (2) shows how authentication and circuit parameters are related:

$$
T_{A u} \propto \frac{N_{T r} \times T_{T e s t e r} \times\left(P_{T H}^{-1}-1\right)}{C_{\text {activity }}}
$$

$T_{A u}$ is a user-defined parameter that depends on time-tomarket and criticality of the application in which the circuit will be used. The equation is based on the time-to-generate a specific number of transitions in a Trojan gate. From Geometric Distribution analysis, on average, $\left(P_{T H}^{-1}-1\right)$ clock cycles are required for each transition on nets whose transition probabilities are $P_{T H}$. It is assumed that the inputs of Trojans are nets with transition probabilities of $P_{T H}$ in the Equation to consider the worst authentication case. $T_{A u}$ is also subjected to $C_{\text {activity }}$ reversely.

Equation (2) shows that there is a direct relation between $T_{A u}, N_{T r}$ and $T_{T e s t e r}$ :

1) Requiring the more number of transitions at Trojan implies the longer authentication time.

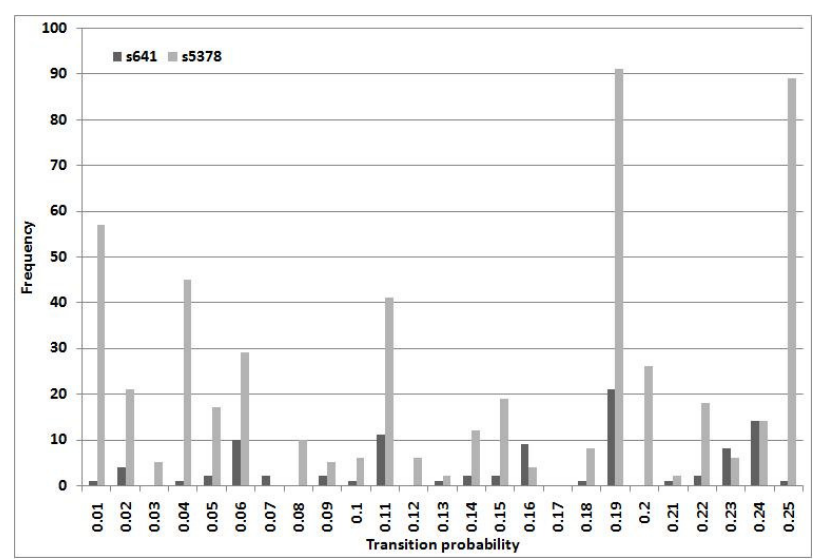

Fig. 10. Transition probability frequency in s641 and s5378 benchmarks.

TABLE III

S641 AND S5378 BENCHMARKS CHARACTERISTICS.

\begin{tabular}{|l||l|l|l|}
\hline Benchmark & \# of inputs & $\begin{array}{l}\text { \# of flip- } \\
\text { flops }\end{array}$ & $\begin{array}{l}\text { the number of compo- } \\
\text { nents }\end{array}$ \\
\hline s5378 & 35 & 176 & 823 \\
\hline s641 & 35 & 19 & 127 \\
\hline
\end{tabular}

2) Clock period of tester $\left(T_{\text {Tester }}\right)$ determines how fast authentication patterns can be applied to IUA. Applying patterns with higher frequency decreases $T_{A u}$.

$P_{T H}$ determines transition probability threshold of design and using Geometric distribution increasing $P_{T H}$ would decrease $T_{A u}$. For a specific design with $C_{\text {activity }}$ of "unit" and assuming $T_{\text {Tester }}=4 \times 10^{-3}$ second, Figure 9 shows that for a target authentication time, $P_{T H}$ increases by the number of required transitions at Trojan output; therefore, area overhead increases. Further, $P_{T H}$ decreases at any specific number of transitions by increasing authentication time. The minimum $P_{T H}$ is obtained when the number of transitions is minimum and authentication time is maximum.

Circuit activity $\left(C_{\text {activity }}\right)$ is a function of transition occurrence frequency of circuit which is defined as

$$
\sum_{P t=0}^{0.25}\left(f_{P t} \times P t \times \sum_{P t^{\prime}=P t}^{0.25}\left(f_{P t^{\prime}} \times P t^{\prime}\right)\right)
$$

where $P t$ and $P t^{\prime}$ is transition probability and $f_{P t}$ and $f_{P t^{\prime}}$ represents the number of nets with transition probability of $P t$ in the entire circuit. Equation (3) represents circuit characteristics by importing the influence of nets with higher transition probabilities on nets with lower transition probabilities. Circuit activity parameter is studied for s5378 and s641 benchmarks by inserting a NAND gate Trojan. Trojan inputs in the two benchmarks have roughly the same transition probabilities $\left(P_{t}=0.015\right)$. Table III shows the benchmarks' characteristics and Figure 10 presents their transition probability frequency. Although both circuits have the same number of inputs, but s5378 is larger with more number of components, consisting of gates and flip-flops. Transition occurrence frequencies of s5378 and s641 benchmarks are 578.84 and 124.92, respectively. It is expected that s5378 with higher $C_{\text {activity }}$ generates more number of transitions in the Trojan. Considering $P_{T H}=$ 
$10 e-05$, simulation results show that there is one transition in each 29.8 clock cycles at the output of Trojan in s5378 whereas 132.0 clock cycles in s641, on average.

$T_{A u}$ depends on $N_{T r}$ and circuit characteristics in terms of $C_{\text {activity }}$ and $P_{T H} . N_{T r}$ determines the number of required transitions to distinguish between Trojan-inserted and Trojanfree circuits. Circuits with higher $C_{\text {activity }}$ may increase Trojan activation and reduce $T_{A u}$. Furthermore, $P_{T H}$ provides and estimation of maximum $T_{A u}$ by implying the rarest Trojan input vector application.

\section{Simulation Results}

We apply our dummy flip-flop insertion procedure to $\mathrm{s} 38417$ benchmark which contains 1564 flip-flops and 4933 gates. Four different transition probability thresholds are examined in this work $\left(P_{T H}=10 \mathrm{e}-05,10 \mathrm{e}-04,10 \mathrm{e}-03\right.$, and 10e-2). The amount of area overhead (number of dSFFs) to ensure all nets have transition probabilities higher than $P_{T H}$ is evaluated. Further, four combinational comparator Trojan circuits, presented in Figure 11, are inserted into the benchmark circuit. Assuming that $P_{T H}=10 \mathrm{e}-05$, transition probabilities of nets are calculated and nets are divided into three groups: (1) Low Transition (LT) nets whose transition probabilities are less than 10e-05, (2) Medium Transition (MT) nets whose transition probabilities are between 10e-05 and 5x10e-05, and (3) High Transition (HT) probability nets whose transition probabilities are greater than $5 \times 10 \mathrm{e}-05$. Similar categorization is used for the other $P_{T H} \mathrm{~s}$ used in this paper. To simulate the worst cases of Trojan activation, nets of the first and second categories are selected to be connected to the Trojans.

Each Trojan circuit consists of two parts: Trigger and Payload. As in Figure 11, the Payload inputs come from Trigger output and data input which is part of the original circuit. The comparators look for rare combinations of Trojan inputs based on their ' 1 ' and ' 0 ' probabilities. Payload gates are selected based on Trojan outputs' dominant values. Dash lines above Trojans in the figure represent the connection in the original circuit which is assumed to be restitched through Trojan's Payload by adversary.

The simulation results show the number of transitions in the entire circuit and specifically transitions of LT and MT nets. The total number of transitions at Trojan inputs and in Trojan circuit, and the number of transitions on Trigger output that can potentially cause functional failure are reported. Then, it is studied how much dSFFs can magnify Trojan impact based on Trojan to Circuit Activity (TCA) factor which is the ratio of the number of transitions in Trojan circuit $\left(N_{T r}\right)$ to the entire design. Additionally, the number of transitions on Payload output is also obtained and we will investigate the difference between payload output and its data input to further analyze the number of erroneous logic values injected into the circuit.

When the value of Trigger output is dormant (i.e. ' 1 ' for AND/NAND Payloads and ' 0 ' for OR/NOR Payloads), the Payload output is the same as Payload's data input; otherwise, the Payload output depends on values of both Trigger output and data input. If both are the same, then the output will be similar to the both inputs. However, a different Payload input combination assuming the Trigger is active would mean that the Payload output is due to Trigger input. This is called full activation of Trojan since the Payload output change (POC) can cause functional failure.

The POC rate depends on transition rate of Trigger output and Payload data input. It is expected when both Payload inputs have low transition probability the POC rate to be unpredictable (small or large). For example, if Payload is an AND gate and data input and Trigger output have high ' 1 ' probability, low POC rate is expected. On the other hand, if one of the Payload inputs has higher transition probability than the other, larger POC rate is expected. If Trigger output is active for many clock cycles, a large Payload output change is expected.

The proposed method can help Trojan detection in two ways:

1. Transient Power Analysis : By increasing the number of transitions in Trojan circuits, the proposed method can help improve the previously proposed power-based methods [5][6][9][11]. In this case, the vectors are applied in a test-per-clock (TPC) fashion since no observation is made by the flip-flops. In fact, the power pads and $\mathrm{C} 4 \mathrm{~s}$ are the observation points since transient current is being measured. Suppose $N_{s f f}$ is the number of scan flip-flops and $N_{v e c_{-} t p c}$ is the number of vectors, the total number of clock cycles $N_{\text {total } y c l e}=N_{v e c}+N_{s f f}-1$. When $N_{v e c} \gg N_{s f f}$, the total number of clock cycles equals the number of test vectors $N_{\text {total_cycle_tpc }}=$ $N_{\text {vec }}$.

2. Full Activation : By increasing the probability of full activation of a Trojan (making the data input to be different from Payload output) the probability of observing an incorrect response to the applied vectors would also increase. In this case, the test vectors are applied in a test-per-scan fashion since the response of a test vector pair must be captured and scanned-out. The test vectors are applied similar to launch-off-shift method used for delay testing except that there is no requirement on atspeed scan enable signal. The second vector is only 1bit shifted version of the first vector (i.e. initialization vector). If $N_{s f f}$ is the number of scan flip-flops and $N_{\text {vec_tps }}$ is the number of vectors, the total number of clock cycles $N_{\text {total_cycle_tps }}=\left(N_{s f f}+1\right) \cdot N_{v e c \_t p s}$.

\section{A. Without Dummy Flip-Flop}

Simulations are run for $N_{v e c_{-} t p c}=N_{\text {vec_tps }}=144$ test vectors. Table IV shows the design transitions statistics and the contribution of Trojans into the original circuit, i.e. before dSFF insertion. Column 2 shows the number of transitions in the entire circuit including Trojans. In the next two columns, transition count for LT and MT nets are reported. These numbers can represent activity of nets which are more probable to constitute Trojans' inputs to make Trojan activations rare. The fifth column presents the number of transitions at 


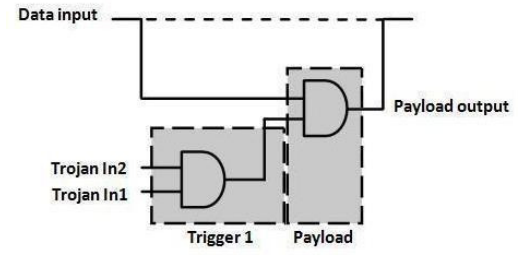

(a) Trojan 1

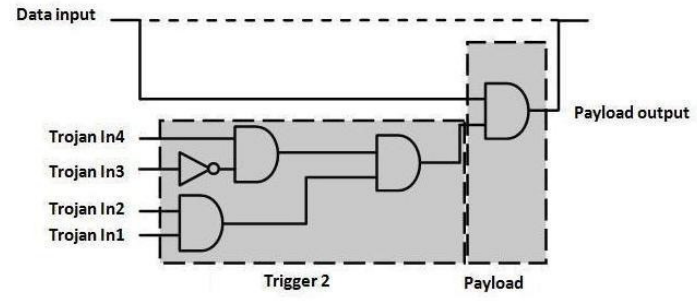

(b) Trojan 2

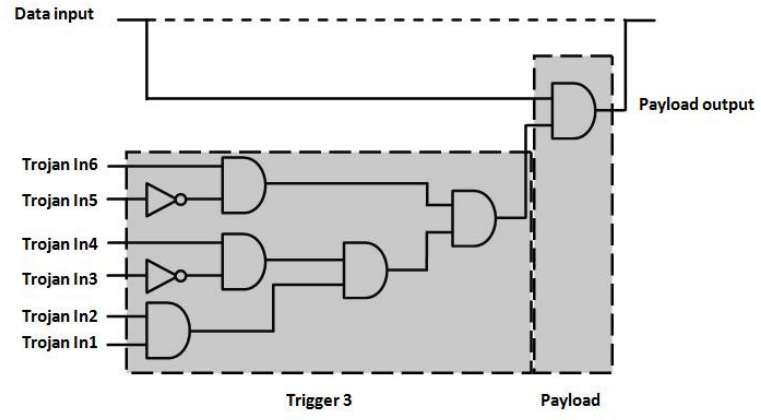

(c) Trojan 3

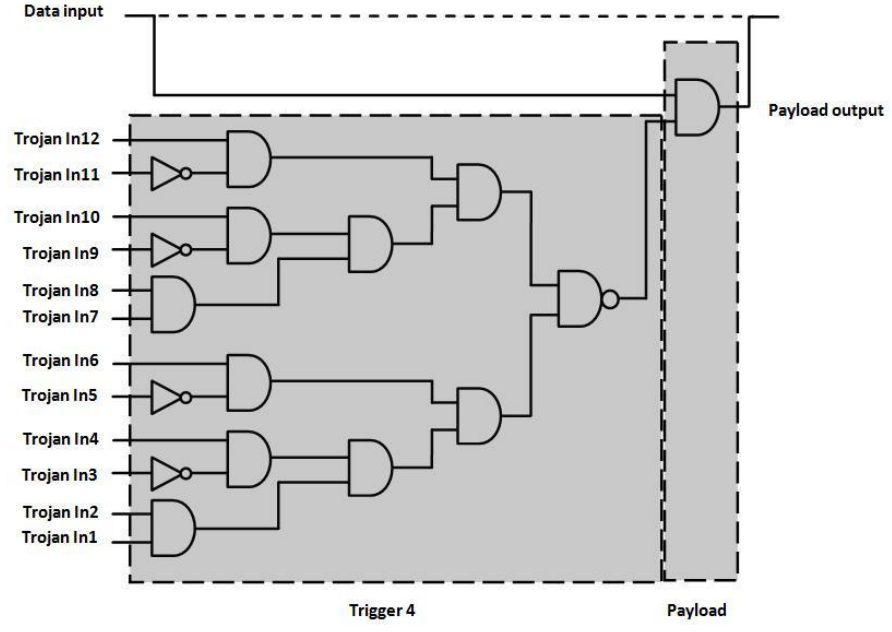

(d) Trojan 4

Fig. 11. Trojan circuits.

TABLE IV

TROJANS ACTIVITY ANALYSIS BEFORE DSFF INSERTION.

\begin{tabular}{|c|c|c|c|c|c|c|c|c|c|}
\hline Trojan & $\begin{array}{l}\text { Total number of } \\
\text { Transitions }\end{array}$ & $\begin{array}{l}\text { \# of Transi- } \\
\text { tions on LT } \\
\text { nets }\end{array}$ & $\begin{array}{l}\text { \# of Transi- } \\
\text { tions on MT } \\
\text { nets }\end{array}$ & $\begin{array}{l}\# \text { of Transitions } \\
\text { at Trojan inputs }\end{array}$ & $\begin{array}{l}\text { \# of Transitions } \\
\text { inside Trojan cir- } \\
\text { cuit }\end{array}$ & $\begin{array}{l}\# \text { of Transitions } \\
\text { at Trojan output }\end{array}$ & $N_{T r}$ & TCA & POC \\
\hline $\begin{array}{l}\text { Trojan } 1 \\
\end{array}$ & 310418 & 7 & 105 & 4 & $\mathrm{NA}^{a}$ & 0 & 0 & $0.0 \mathrm{E}+00$ & 0 \\
\hline Trojan 2 & 310429 & 7 & 105 & 16 & 11 & 0 & 11 & $3.54 \mathrm{E}-05$ & 0 \\
\hline Trojan 3 & 310443 & 7 & 105 & 35 & 25 & 0 & 25 & $8.05 \mathrm{E}-05$ & 0 \\
\hline Trojan 4 & 310470 & 7 & 105 & 89 & 51 & 0 & 51 & $1.64 \mathrm{E}-04$ & 0 \\
\hline
\end{tabular}

${ }^{a}$ Not Applicable

TABLE V

Trojans ACTIVITy ANALYSIS AFTER DSFF INSERTION with $P_{T H}=10 e-5$.

\begin{tabular}{|c|c|c|c|c|c|c|c|c|c|}
\hline Trojan & $\begin{array}{l}\text { Total number of } \\
\text { Transitions }\end{array}$ & $\begin{array}{l}\text { \# of Transi- } \\
\text { tions on LT } \\
\text { nets }\end{array}$ & $\begin{array}{l}\text { \# of Transi- } \\
\text { tions on MT } \\
\text { nets }\end{array}$ & $\begin{array}{l}\# \text { of Transitions } \\
\text { at Trojan inputs }\end{array}$ & $\begin{array}{l}\text { \# of Transitions } \\
\text { inside Trojan cir- } \\
\text { cuit }\end{array}$ & $\begin{array}{l}\# \text { of Transitions } \\
\text { at Trojan output }\end{array}$ & $N_{T r}$ & TCA & POC \\
\hline Trojan 1 & 318257 & 57 & 144 & 37 & NA & 1 & 1 & $3.13 \mathrm{E}-06$ & 0 \\
\hline Trojan 2 & 318284 & 57 & 144 & 64 & 14 & 0 & 14 & $4.39 \mathrm{E}-05$ & 0 \\
\hline Trojan 3 & 318299 & 57 & 144 & 97 & 28 & 0 & 28 & $8.79 \mathrm{E}-05$ & 0 \\
\hline Trojan 4 & 318365 & 57 & 144 & 163 & 95 & 0 & 95 & $2.98 \mathrm{E}-04$ & 0 \\
\hline
\end{tabular}

Trojan inputs, implying attempts to activate Trojans subjected to various input combinations. Columns 6 and 7 show the number of transitions inside and at the output of Trojans, respectively. The total number of transitions in Trojans $\left(N_{T r}\right)$, the sum of transitions inside and at the output of Trojans, is reported in Column 8. Trojan contribution into the entire circuit is evaluated by TCA factor and presented in Column 9. The last column (POC) indicates the number of Trojan full activations which results in functional error inside the host design.

Table IV shows that before dSFF insertion none of the Trojans is fully activated. The results indicate larger Trojans 
TABLE VI

Trojans ACTIVITY ANALYSIS AFTER DSFF INSERTION WITH $P_{T H}=10 e-4$.

\begin{tabular}{|c|c|c|c|c|c|c|c|c|c|}
\hline Trojan & $\begin{array}{l}\text { Total number of } \\
\text { Transitions }\end{array}$ & $\begin{array}{l}\text { \# of Transi- } \\
\text { tions on LT } \\
\text { nets }\end{array}$ & $\begin{array}{l}\text { \# of Transi- } \\
\text { tions on MT } \\
\text { nets }\end{array}$ & $\begin{array}{l}\text { \# of Transitions } \\
\text { at Trojan inputs }\end{array}$ & $\begin{array}{l}\# \text { of Transitions } \\
\text { inside Trojan cir- } \\
\text { cuit }\end{array}$ & $\begin{array}{l}\text { of Transitions } \\
\text { at Trojan output }\end{array}$ & $N_{T r}$ & TCA & POC \\
\hline $\begin{array}{l}\text { Trojan } 1 \\
\end{array}$ & 327293 & 149 & 399 & 69 & NA & 18 & 18 & $5.49 \mathrm{E}-05$ & 14 \\
\hline Trojan 2 & 327322 & 149 & 399 & 148 & 73 & 7 & 80 & $2.44 \mathrm{E}-04$ & 9 \\
\hline Trojan 3 & 327357 & 149 & 399 & 190 & 116 & 0 & 116 & $3.54 \mathrm{E}-04$ & 6 \\
\hline Trojan 4 & 327436 & 149 & 399 & 361 & 195 & 0 & 195 & $5.95 \mathrm{E}-04$ & 0 \\
\hline
\end{tabular}

TABLE VII

TROJANS ACTIVITY ANALYSIS AFTER DSFF INSERTION WITH $P_{T H}=10 e-3$.

\begin{tabular}{|c|c|c|c|c|c|c|c|c|c|}
\hline Trojan & $\begin{array}{l}\text { Total number of } \\
\text { Transitions }\end{array}$ & $\begin{array}{l}\text { \# of Transi- } \\
\text { tions on LT } \\
\text { nets }\end{array}$ & $\begin{array}{l}\text { \# of Transi- } \\
\text { tions on MT } \\
\text { nets }\end{array}$ & $\begin{array}{l}\# \text { of Transitions } \\
\text { at Trojan inputs }\end{array}$ & $\begin{array}{l}\# \text { of Transitions } \\
\text { inside Trojan cir- } \\
\text { cuit }\end{array}$ & $\begin{array}{l}\# \text { of Transitions } \\
\text { at Trojan output }\end{array}$ & $N_{T r}$ & TCA & $\overline{P O C}$ \\
\hline$\overline{\overline{\text { Trojan } 1}}$ & 2235378 & $\bar{~} 46$ & 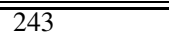 & 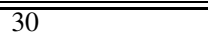 & $\overline{\mathrm{NA}}$ & 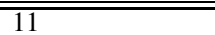 & 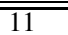 & 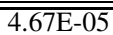 & 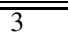 \\
\hline Trojan 2 & 235394 & 46 & 243 & 42 & 38 & 1 & 39 & $1.65 \mathrm{E}-04$ & 0 \\
\hline Trojan 3 & 235418 & 46 & 243 & 96 & 62 & 0 & 62 & $2.63 \mathrm{E}-04$ & 0 \\
\hline Trojan 4 & 235523 & 46 & 243 & 186 & 167 & 0 & 167 & $7.09 \mathrm{E}-04$ & 0 \\
\hline
\end{tabular}

contribute more into the entire design activity, i.e. larger $N_{T r}$, and thus have greater TCA, more attributed to internal Transitions.

\section{B. $P_{T H}=10 e-05$}

There are four nets in $\mathrm{s} 38417$ benchmark with transition probability less than $10 \mathrm{e}-05$. Using our procedure, 4 dSFFs are needed to increase transition probabilities of these nets beyond 10e-05. The 4 dSFFs make an area overhead about $0.2 \%$. Table $\mathrm{V}$ shows circuit activity decreases slightly while Trojans activity increase. Although none of the Trojans is fully activated, there is increase in Trojans' TCA in proportion to their size. Furthermore, activity of LT and MT nets increases and is manifested in increasing Trojans' activity. In the following, $P_{T H}$ is increased to $10 \mathrm{e}-04$ and corresponding results are presented in Table VI.

\section{C. $P_{T H}=10 e-04$}

The dSFF insertion procedure identifies 28 nets with transition probability less than $10 \mathrm{e}-04$. In this case, $16 \mathrm{dSFFs}$ are inserted to ensure these nets have transition probability greater than $P_{T H}$, incurring $0.8 \%$ area overhead. The results in Table VI show LT and MT nets are more active by increasing $P_{T H}$ relative to the previous cases. In addition, Trojans of smaller size are fully activated and cause functional errors in the host design several times, and larger Trojans bring forth more internal transitions. Consequently, there is high activity in Trojan circuits and significant increase in Trojans' TCA. To verify that continuously increasing $P_{T H}$ increases Trojans contribution, $P_{T H}$ is increased to $10 \mathrm{e}-03$ and

\section{D. $P_{T H}=10 e-03$}

Increasing transition probability of nets beyond $10 \mathrm{e}-03$ requires $60 \mathrm{dSFFs}$ and imposes $3.0 \%$ overhead. The results in Table VII, contrary to what was expected, show decrease in both circuit and Trojans activity. LT and MT nets are less active relative to $P_{T H}=10 \mathrm{e}-04$, and as a result Trojans get less activated. Even the total number of transitions in the entire design decreases. As an exception the TCA factor of Trojan 4 is increased although it gets less active due to relatively more decrease in the total number of transitions in the entire design. More detail analysis in the following shows that increasing transition probability of nets beyond a specific threshold does not necessarily increase the number of transitions in the entire design.

Any circuit consists of primary gates mainly NAND and NOR gates, and any other complex gate and module can be made using these primary gates. Transition at the output of a gate is a function of transition on its inputs. Figure 12 shows transition probability at the output of 2-input NAND and 2input NOR gates based on transition probability of their inputs. The maximum transition probability of a net is 0.25 and is obtained when probabilities of 1 and 0 of the net are equal to 1/2. However, Figure 12 indicates that maximum transition probability at the output of the gates are when transition probability of one of its input is high and that of the other input is low. This trend can be seen in both NAND and NOR gates. Further, Figure 12 indicates when transition probabilities of inputs are both 0.25 (the maximum value), the transition probabilities at gates' outputs are 0.1875 in the both gates. In sum, increasing $P_{T H}$ to increase transition probability of individual nets may not necessarily increase the number of transitions in the entire design. To confirm this fact $P_{T H}$ is increased to $10 \mathrm{e}-02$ and results are presented in Table VIII.

\section{E. $P_{T H}=10 e-02$}

Incurring 5.2\% area overhead, $100 \mathrm{dSFFs}$ are required to have transition probability of all nets greater than $10 \mathrm{e}-02$. As it was expected the total number of transitions in the entire design decreases more and the number of transition inside and at the output of Trojan circuits is less than the results with $P_{T H}=10 \mathrm{e}-04$. However, since the number of transitions in the entire design is roughly half of corresponding values with $P_{T H}=10 \mathrm{e}-04$ there is increase in Trojans' TCA with $P_{T H}=$ 10e-02. 

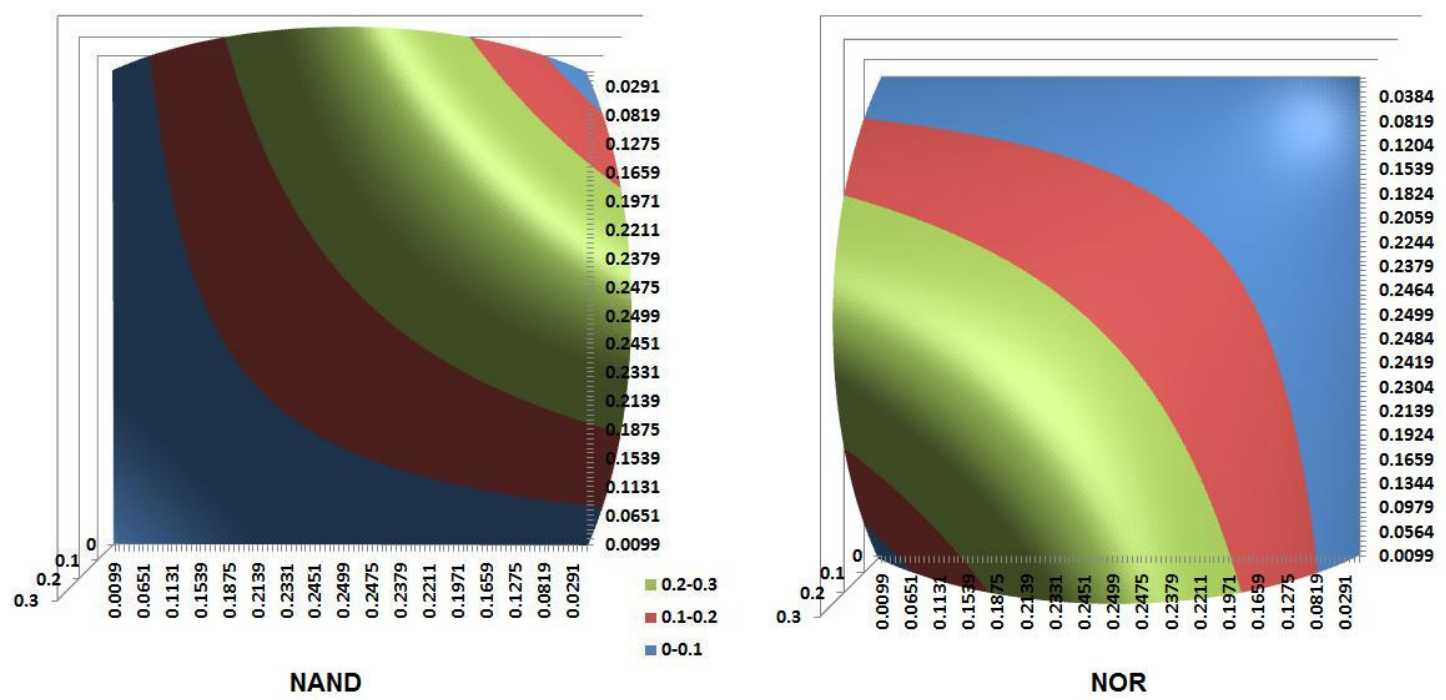

Fig. 12. Transition density analysis for NAND and NOR gates.

TABLE VIII

Trojans aCtivity ANALYSIS AFTER DSFF INSERTION WITH $P_{T H}=10 e-2$.

\begin{tabular}{|c|c|c|c|c|c|c|c|c|c|}
\hline Trojan & $\begin{array}{l}\text { Total number of } \\
\text { Transitions }\end{array}$ & $\begin{array}{l}\text { \# of Transi- } \\
\text { tions on LT } \\
\text { nets }\end{array}$ & $\begin{array}{l}\text { \# of Transi- } \\
\text { tions on MT } \\
\text { nets }\end{array}$ & $\begin{array}{l}\# \text { of Transitions } \\
\text { at Trojan inputs }\end{array}$ & $\begin{array}{l}\# \text { of Transitions } \\
\text { inside Trojan cir- } \\
\text { cuit }\end{array}$ & $\begin{array}{l}\# \text { of Transitions } \\
\text { at Trojan output }\end{array}$ & $N_{T r}$ & TCA & POC \\
\hline "Trojan 1 & $\bar{~} 185875$ & $\overline{1399}$ & 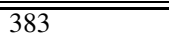 & $\overline{c 67}$ & 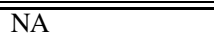 & 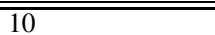 & $\overline{10}$ & $\overline{c 5.38 \mathrm{E}-05}$ & $\overline{\overline{5}}$ \\
\hline Trojan 2 & 185915 & 139 & 383 & 132 & 67 & 2 & 69 & $3.71 \mathrm{E}-04$ & 0 \\
\hline Trojan 3 & 185946 & 139 & 383 & 176 & 100 & 0 & 100 & $5.37 \mathrm{E}-04$ & 0 \\
\hline Trojan 4 & 186049 & 139 & 383 & 352 & 204 & 0 & 204 & 1.09E-03 & 0 \\
\hline
\end{tabular}

TABLE IX

THE TRANSITIONS STATISTICS IN THE ENTIRE DESIGN AND MT AND LT NETS.

\begin{tabular}{|l||l|l|l|l|l|}
\hline Pth & $\begin{array}{l}\text { The number of transitions } \\
\text { in the entire design }\end{array}$ & $\begin{array}{l}\text { The transitions percent- } \\
\text { age of MT and LT nets }\end{array}$ & $\begin{array}{l}\text { Average number of } \\
\text { transitions on LT and } \\
\text { MT nets }\end{array}$ & $\begin{array}{l}\text { Average number of } \\
\text { transitions per clock } \\
\text { in the entire design }\end{array}$ & $\begin{array}{l}\text { Average number of } \\
\text { transitions on each net }\end{array}$ \\
\hline Before dSFF insertion & 310418 & $3.6 \mathrm{e}-02 \%$ & 5.6 & 2155 & 6 \\
\hline $10 \mathrm{e}-05$ & 318527 & $6.3 \mathrm{e}-02 \%$ & 10.0 & 2212 & 66 \\
\hline $10 \mathrm{e}-04$ & $1.5 \mathrm{e}-01 \%$ & 25.4 & 2283 & 70 \\
\hline $10 \mathrm{e}-03$ & $1.2 \mathrm{e}-01 \%$ & 14.4 & 1641 & 50 \\
\hline $10 \mathrm{e}-02$ & 328684 & $2.8 \mathrm{e}-01 \%$ & 26.1 & 1290 & 39 \\
\hline
\end{tabular}

Table IX presents transitions statistics in the entire design and LT and MT nets at examined $P_{T H}$ s. The results in Column 2 indicate increasing $P_{T H}$ decreases the number of transitions in the entire design, caused by transition characteristics of primary gates discussed. On the other hand, Column 3 shows increase in the percentage of transitions on LT and MT nets. In other words, there is a transition movement from HT nets to MT and LT nets. The next column also demonstrates that by increasing $P_{T H}$, the average number of transitions on LT and MT nets would increase. The last two columns corroborate that by increasing $P_{T H}$ there is decrease in the number of transitions in the entire design per clock and on each net on average.

In Table $\mathrm{X}$, the results show that by increasing $P_{T H}$ although there are more number of low transition nets, relatively there is decrease in the number of required dSFF. Further, the simulation results show that smaller Trojans, e.g. Trojan 1 and Trojan 2, can be fully activated with higher rate while they have less contribution into circuit activity. On the other
TABLE X

$P_{T H}$ ANALYSIS.

\begin{tabular}{|c||c|c|c|c|}
\hline Pth & $10 \mathrm{e}-05$ & $10 \mathrm{e}-04$ & $10 \mathrm{e}-03$ & $10 \mathrm{e}-02$ \\
\hline the number of nets & 4 & 28 & 129 & 275 \\
\hline the number of dSFFs & 4 & 16 & 60 & 100 \\
\hline Area overhead & $0.2 \%$ & $0.8 \%$ & $3.0 \%$ & $5.2 \%$ \\
\hline The ratio of \# dSFF to \# nets & $100 \%$ & $57 \%$ & $46 \%$ & $36 \%$ \\
\hline
\end{tabular}

hand, larger Trojans, e.g. Trojan 3 and Trojan 4, are harder to be fully activated and contribute more into circuit activity. Therefore, we believe that smaller Trojans are easier to detect using ATPG with dSFF insertion technique and larger Trojans using power-based techniques.

\section{CONCLUSIONS}

In this paper, we demonstrate that the topology of a circuit and the number of primary inputs and flip-flops determine switching activity of the circuit. In the following, transitions are modeled using geometric distribution and the number of clock cycles taking to generate a transition is estimated on 
average. Furthermore, it is shown that inserting dummy scan flip-flop can reduce transition generating time. This realization leads to develop a dummy flip-flop insertion procedure aiming at augmenting transition probabilities of nets in a design, and increasing activity of hardware Trojans in Integrated Circuits. The simulation results for $\mathrm{s} 38417$ benchmark demonstrate that it is possible to significantly increase switching activity in Trojan circuits. Smaller Trojans may be fully activated and cause functional failures. Larger Trojans more contribute into side-channel signals and are detected as abnormality.

\section{ACKNOWLEDGEMENTS}

The work of Hassan Salmani and Mohammad Tehranipoor was supported in part by the National Science Foundation Grant CNS-0716535. The work of Jim Plusquellic was supported in part by NSF grant CNS-0716559.

\section{REFERENCES}

[1] U.S.D. Of Defense. "Defense science board task force on high performance microchip supply," http://www.acq.osd.mil/dsb/reports/2005-02HPMS_Report_Final.pdf

[2] S. Adee "The Hunt for the Kill Switch," http://www.spectrum.ieee.org/print/6171

[3] X. Wang, M. Tehranipoor and J. Plusquellic, "Detecting malicious inclusions in secure hardware: Challenges and solutions," in Proc. of the IEEE International Workshop on Hardware-Oriented Security and Trust(HOST 2008), pp. 15-19, 2008.

[4] M. Banga and M. S. Hsiao, "A Novel Sustained Vector Technique for the Detection of Hardware Trojans," in Proc. of the International Conference on VLSI Design, pp. 327-332, 2009.

[5] R. S. Chakraborty and S. Bhunia, "Security Against Hardware Trojan Through a Novel Application of Design Obfuscation," in Proc. of the International Conference on Computer-aided Design(ICCAD09), 2009.

[6] M. Banga and M. S. Hsiao, "A region based approach for the identification of hardware trojans," in Proc. of the IEEE International Workshop on Hardware-Oriented Security and Trust (HOST08), pp. 40-47, June 2008.

[7] M. Banga, M. Chandrasekar, L. Fang, and M. S. Hsiao, "Guided test generation for isolation and detection of embedded trojans in ICs," in Proc. of the in Proceedings of the IEEE/ACM Great Lakes Symposium on VLSI, pp. 363-366, April 2008.

[8] D. Agrawal, S. Baktir, D. Karakoyunlu, P. Rohatgi and B. Sunar, "Trojan Detection using IC Fingerprinting," in Proc. of the Symposium on Security and Privacy, pp. 296-310, 2007.

[9] R. Rad, X. Wang, J. Plusquellic and M. Tehranipoor, "Power Supply Signal Calibration Techniques for Improving Detection Resolution to Hardware Trojans," in Proc. of the International Conference on Computer-Aided Design (ICCAD08), pp. 632-639, 2008.

[10] J. Li and J. Lach, "At-speed delay characterization for IC authentication and Trojan Horse detection," in Proc. of the IEEE International Workshop on Hardware-Oriented Security and Trust(HOST 2008), pp. 8-14, 2008.

[11] Y. Jin and Y. Makris, "Hardware Trojan detection using path delay fingerprint," in Proc. of the IEEE International Workshop on HardwareOriented Security and Trust(HOST 2008), pp. 51-57, 2008.

[12] D. D. Wackerly, W. Mendenhall III and R. L. Scheaffer, "Mathematical Statistics with Application, 7th edition" Thomson Learning, Inc., 2008.

[13] X. Wang, H. Salmani, M. Tehranipoor and J. Plusquellic, "Hardware Trojan Detection and Isolation Using Current Integration and Localized Current Analysis," in Proc. of the International Symposium on Fault and Defect Tolerance in VLSI Systems (DFT08), pp. 87-95, 2008.

[14] M. Bushnell and V. Agrawal, "Essentials of Electronics Testing," Kluwer Publishers, 2000.

[15] S. Jha and S. K. Jha, "Randomization Based Probabilistic Approach to Detect Trojan Circuits," in Proc. of the IEEE High Assurance Systems Engineering Symposium(HASE08), pp. 117-124, 2008.

[16] M. Potkonjak, A. Nahapetian, M. Nelson, T. Massey, "Hardware Trojan Horse Detection Using Gate-Level Characterization," in Proc. of the IEEE International Design Automation Conference (DAC), 2009
[17] Y. Alkabani, F. Koushanfar, "Consistency-Based Characterization for IC Trojan Detection," in Proc. of the International Conference on Computer-Aided Design (ICCAD09), 2009.

[18] F. Wolff, C. Papachristou, S. Bhunia and R.S. Chakraborty, "Towards Trojan-Free Trusted ICs: Problem Analysis and Detection Scheme," in Proc. of the Design, Automation and Test in Europe(DATE '08), pp. 1362-1365, 2008.

[19] R. S. Chakraborty, F. Wolff, S. Paul, C. Papachristou and S. Bhunia, "MERO: A Statistical Approach for Hardware Trojan Detection," in Proc. of the Workshop on Cryptographic Hardware and Embedded Systems (CHES), 2009.

[20] R. Sankaralingam, R. R. Oruganti and N. A. Touba, "Static Compaction Techniques to Control Scan Vector Power Dissipation," in Proc. of the IEEE VLSI Test Symposium (VTS'00), pp. 35-40, 2000. 